\title{
The VLPFC versus the DLPFC in Downregulating Social Pain Using Reappraisal and Distraction Strategies
}

\author{
Jun Zhao, ${ }^{1,2 *}$ Licheng Mo, ${ }^{1 *}$ Rong Bi, ${ }^{1}$ Zhenhong He, ${ }^{1}$ Yuming Chen, ${ }^{1}$ Feng $\mathrm{Xu},{ }^{3}$ Hui Xie, ${ }^{1}$ and \\ Dandan Zhang ${ }^{1,2,4,5}$ \\ ${ }^{1}$ School of Psychology, Shenzhen University, Shenzhen 518060, China, ${ }^{2}$ Institute of Brain and Psychological Sciences, Sichuan Normal University, \\ Chengdu 610066, China, ${ }^{3}$ Shenzhen Yingchi Technology Company, Ltd, Shenzhen 518057, China, ${ }^{4}$ Shenzhen-Hong Kong Institute of Brain Science, \\ Shenzhen 518060, China, and ${ }^{5}$ Shenzhen Institute of Neuroscience, Shenzhen 518060, China
}

The dorsolateral prefrontal cortex (DLPFC) and ventrolateral PFC (VLPFC) are both crucial structures involved in voluntary emotional regulation. However, it remains unclear whether the functions of these two cortical regions that are involved in emotional regulation, which are usually active in non-social situations, could be generalized to the regulation of social pain as well. This study employed transcranial magnetic stimulation (TMS) to examine the causal relationship between the DLPFC/VLPFC and the emotional regulation of social pain via distraction and reappraisal. Ninety human participants (45 males and 45 females) initially underwent either active (DLPFC/VLPFC, $n=30 / 30$ ) or sham (vertex, $n=30$ ) TMS sessions. Participants were then instructed to use both distraction and reappraisal strategies to downregulate any negative emotions evoked by social exclusion pictures. Convergent results of the subjective emotional rating and electrophysiological indices demonstrated that: (1) both the DLPFC and VLPFC highly facilitate the downregulation of affective responses caused by social exclusion, revealing a causal role of these lateral PFCs in voluntary emotional regulation of both non-social and social pain; and (2) these two cortical regions showed relative functional specificity for distraction (DLPFC) and reappraisal (VLPFC) strategies, which helps to refine the cortical targeting of therapeutic protocols. In addition, the TMS effect was sustainable for at least $1 \mathrm{~h}$, showcasing the potential feasibility of using this method in clinical practice. Together, these findings provide cognitive and neural evidence for the targeting of the VLPFC and/or the DLPFC to improve emotional regulation abilities, especially in social contexts.

Key words: dorsolateral prefrontal cortex; emotional regulation; social exclusion; social pain; TMS; ventrolateral prefrontal cortex

\section{Significance Statement}

This study aimed to examine the role of the dorsolateral prefrontal cortex (DLPFC) and ventrolateral PFC (VLPFC) in emotional regulation, particularly in response to social pain through the use of distraction and reappraisal strategies, as this is a relatively underexplored area of inquiry. This study makes a significant contribution to the literature because our results provide novel empirical information on the role of these cortical structures in the processing of negative emotions elicited within certain social contexts. As such, our findings have potential clinical implications, paving the way for future clinicians to be able to accurately target specific brain regions among patients struggling with impaired social cognition abilities, including those diagnosed with posttraumatic stress disorder, autism spectrum disorder, social anxiety disorder, and depression.

Received July 22, 2020; revised 0ct. 21, 2020; accepted 0ct. 23, 2020.

Author contributions: J.Z., Z.H., and D.Z. designed research; J.Z., L.M., and Y.C. performed research; F.X. contributed unpublished reagents/analytic tools; J.Z., L.M., and D.Z. analyzed data; R.B., Z.H., H.X., and D.Z. wrote the paper.

This work was supported by the National Natural Science Foundation of China Grant 31970980, the Shenzhen Basic Research Project JCYJ20180305124305294, the Guangdong Key Basic Research Grant 2018B030332001, and the Shenzhen-Hong Kong Institute of Brain Science Grant 2019 SHIBS0003.

*J.Z. and L.M. contributed equally to this work.

The authors declare no competing financial interests.

Correspondence should be addressed to Dandan Zhang at zhangdd05@gmail.com.

https://doi.org/10.1523/JNEUROSCI.1906-20.2020

Copyright (C) 2021 Zhao, Mo et al.

This is an open-access article distributed under the terms of the Creative Commons Attribution 4.0 International license, which permits unrestricted use, distribution and reproduction in any medium provided that the original work is properly attributed.

\section{Introduction}

While the key brain regions for emotional regulation are primarily located in the prefrontal cortex (PFC; Ochsner et al., 2012), some studies have demonstrated that explicit emotional regulation using varying strategies do not involve the exact overlapping neural substrates (Morawetz et al., 2017; Vrtička et al., 2011). In particular, although the dorsolateral PFC (DLPFC) and ventrolateral PFC (VLPFC) are both important in distraction and reappraisal (Buhle et al., 2014; Kohn et al., 2014), e.g., the two regions often activate together during reappraisal (Ochsner et al., 2012; Morawetz et al., 2017), neuroimaging studies have revealed that while the VLPFC is consistently involved during reappraisal, the 
DLPFC is more associated with distraction (Dörfel et al., 2014; Moodie et al., 2020). However, it remains unclear to what extent these two brain regions are essential and specific for both of these strategies. This study employed transcranial magnetic stimulation (TMS) to examine the roles of the VLPFC and the DLPFC in emotional regulation via distraction and reappraisal strategies.

Meanwhile, a more urgent question is whether the brain regions involved in emotional regulation observed in general, especially non-social, situations can be generalized to functioning similarly in social contexts. Most previous studies have not disentangled the specific brain regions involved in emotional regulation for social versus non-social emotion-eliciting events (but see Vrtička et al., 2011). Uncovering the neural substrates critical for emotional regulation in social contexts is necessary not only for understanding the neural mechanisms behind emotional regulation, but also in the development of effective therapeutic protocols in clinics. For one thing, negative social experiences, including dysfunctional family interactions and traumatic or stressful interpersonal events, are all considered major risk factors in the development of psychiatric disorders such as posttraumatic stress disorder, social anxiety, depression, and autism spectrum disorder (Nolan et al., 2003; Durodié and Wainwright, 2019). For another, growing evidence indicates that these aforementioned psychiatric disorders are all associated with the maladaptive regulation of social pain (Davey et al., 2011; Masten et al., 2011). Therefore, it is implied that improving one's emotional regulation abilities in response to negative social events is highly beneficial for the remission of symptoms and recovery from psychiatric disorders (Laceulle et al., 2017). This study was designed to examine the causal relationship between the VLPFC/DLPFC and the emotional regulation of social pain to provide neural targets for clinical interventions.

Previous studies have demonstrated that both the VLPFC and the DLPFC are associated closely with the reduction of social pain (Koban et al., 2017a; Vijayakumar et al., 2017; Wang et al., 2017). Typically, researchers have observed that the right or bilateral VLPFC is activated significantly when individuals experienced social exclusion (Eisenberger et al., 2003; Masten et al., 2009; Onoda et al., 2010; Hooker et al., 2010; Riva et al., 2012, 2015; Hsu et al., 2015). Also, fMRI studies have found that enhanced activation in the DLPFC was associated with a decline in subjective social distress (Nishiyama et al., 2015; Koban et al., 2017b) and aggressive behaviors (Achterberg et al., 2016, 2020) following the experience of social rejection. However, while these studies have demonstrated an association between the DLPFC/ VLPFC and reduced social pain, very limited studies have examined the role of these regions on explicit emotional regulation within social contexts (but see Koenigsberg et al., 2010). Recently, we used tDCS/TMS in an explicit emotional regulation task and provided direct evidence for the role of the right VLPFC on emotional regulation following the experience of social exclusion ( $\mathrm{He}$ et al., 2018, 2020a,b). However, until now, no study has explored the causal role of the DLPFC in the explicit regulation of social pain. This study was thus inspired by this literature gap.

Our hypothesis is two-fold. First, the critical role of the VLPFC and the DLPFC in emotional regulation during nonsocial situations is generalizable to social ones, because previous studies have revealed a close association between the VLPFC/ DLPFC and reduced social pain. Second, because the VLPFC is consistently involved during reappraisal and the DLPFC is always associated with distraction, we hypothesized that these two regions would show functional segregation, to some extent, for reappraisal and distraction strategies, respectively.
Table 1. Demographical characteristics of the three groups (mean \pm SD)

\begin{tabular}{lllllll}
\hline Items & $\begin{array}{l}\text { VLPFC group } \\
(n=30)\end{array}$ & $\begin{array}{l}\text { DLPFC group } \\
(n=30)\end{array}$ & $\begin{array}{l}\text { Sham group } \\
(n=30)\end{array}$ & \multicolumn{2}{l}{ Statistics $^{\mathrm{a}}$} \\
\cline { 6 - 7 } & Gender (male/female) & $15 / 15$ & $15 / 15$ & $15 / 15$ & $p$ \\
Age (year) & $19.8 \pm 1.6$ & $19.2 \pm 1.4$ & $20.0 \pm 1.8$ & 1.71 & 0.186 \\
STAI-T & $40.8 \pm 8.3$ & $42.6 \pm 10.9$ & $39.1 \pm 9.3$ & 1.02 & 0.363 \\
LSAS & $36.5 \pm 18.3$ & $36.4 \pm 17.6$ & $38.4 \pm 19.6$ & 0.11 & 0.893 \\
BDI-II & $5.6 \pm 4.1$ & $6.9 \pm 6.4$ & $7.3 \pm 7.1$ & 0.66 & 0.522 \\
RSQ & $7.8 \pm 3.7$ & $7.8 \pm 2.8$ & $7.3 \pm 3.6$ & 0.26 & 0.775 \\
IRI & $52.7 \pm 12.3$ & $54.4 \pm 10.3$ & $53.7 \pm 8.6$ & 0.18 & 0.832 \\
\hline
\end{tabular}

${ }^{a}$ One-way ANOVA across the three groups.

STAI-T, the Trait form of Spielberger's State-Trait Anxiety Inventory; LSAS, the Liebowitz Social Anxiety Scale; BDI-II, the Beck Depression Inventory Second Edition; RSQ, the Rejection Sensitivity Questionnaire; IRI, the Interpersonal Reactivity Index.

\section{Materials and Methods}

Participants

This study used three TMS groups: the VLPFC-activated group, the DLPFC-activated group, and the vertex-activated (sham) group. During the experiment design, we conducted a priori power analysis using G*Power 3.1.7 ( $F$ tests, ANOVA: repeated measures, within-between interaction) based on the effect size $\left(\eta_{\mathrm{p}}^{2}=0.130\right)$ reported in our previous TMS study (He et al., 2020a). According to the result of this power analysis, 18 participants in total would ensure $80 \%$ statistical power. However, six participants per group is such a small sample size in present-day neuroscience studies focusing on non-patient population. Thus, we finally decided to include 30 participants per TMS group, the same sample size as in our previous TMS study (He et al., 2020a), which ensured a statistical power near $100 \%$. In line with our previous TMS study (He et al., 2020a), we decided to include 30 participants in each group. Therefore, a total of 90 healthy college students (all right-handed) were recruited from Shenzhen University. They completed five questionnaires before their group assignment, including the Trait form of Spielberger's State-Trait Anxiety Inventory (STAI-T; Spielberger et al., 1983), the Liebowitz Social Anxiety Scale (LSAS; Liebowitz, 1987), the Beck Depression Inventory Second Edition (BDI-II; Beck et al., 1996), the Rejection Sensitivity Questionnaire (RSQ; Downey and Feldman, 1996), and the Interpersonal Reactivity Index (IRI; Davis, 1980). The group assignments counterbalanced the scores of these questionnaires, with participants being assigned with equal numbers of males and females into the three TMS groups. No participant had any prior experiences with TMS before this experiment. No significant differences were found in participants' ages, or in their anxiety scores (STAI-T), social anxious levels (LSAS), depressive tendencies (BDI-II), rejection sensitivities (RSQ), or empathy (IRI) across all three groups (Table 1). The study protocol was approved by the Ethics Committee of Shenzhen University. Informed consent was signed by the participants before their engagement in the experiment.

\section{Materials and experimental procedure}

The experimental materials were 90 social exclusion pictures that were also used in our previous studies (He et al., 2018, 2020a,b). During the experiment, the images were presented in the center of an LCD screen with a viewing angle of $3.0 \times 3.5^{\circ}$

The study was a three (regulation type: no-regulation, reappraisal, and distraction) by three (TMS group: VLPFC-activated, DLPFC-activated, and sham) design. The regulation type was the within-subject factor and the TMS group was the between-subject factor. The task was divided into three blocks, corresponding to the three regulation types. To avoid any carry-over effects caused by the reappraisal/distraction instructions, the passive viewing (i.e., no-regulation) task was always performed first (see also He et al., 2018, 2020a,b). The 90 images were randomly assigned across the three blocks, with each block containing 30 images in total. The order of the three blocks was equal across the three TMS groups, while the order of the two emotional regulation blocks (reappraisal and distraction) was counterbalanced within each TMS group. 


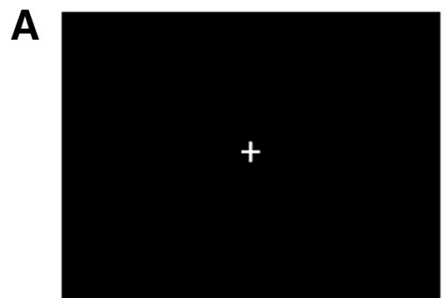

preparation (2s)

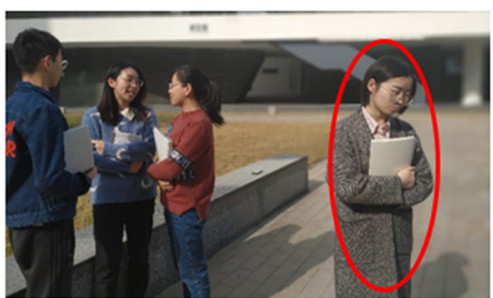

no-regulation/reappraisal/ distraction $(8 \mathrm{~s})$

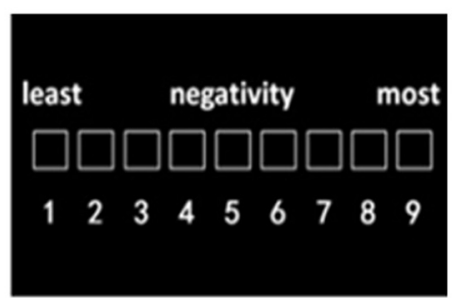

emotion rating $(5 s)$
B

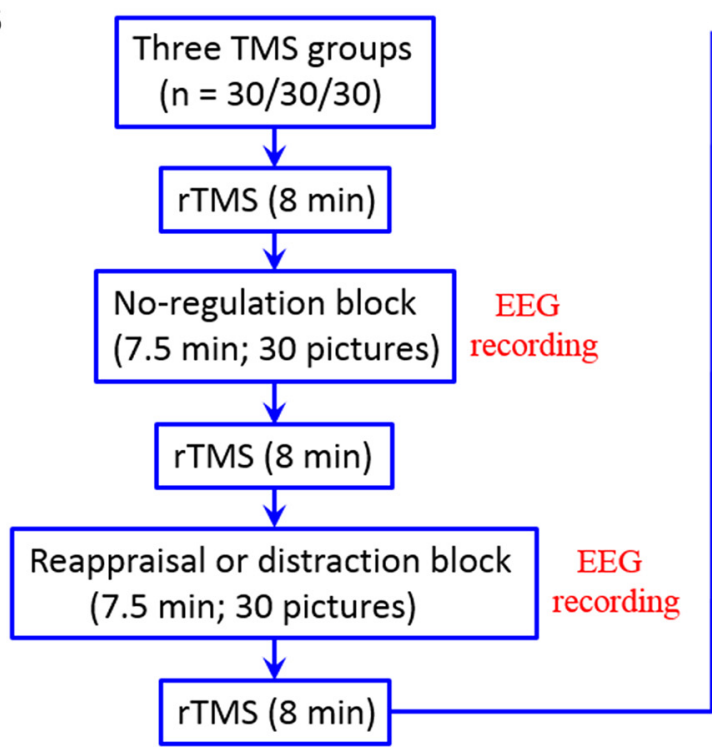

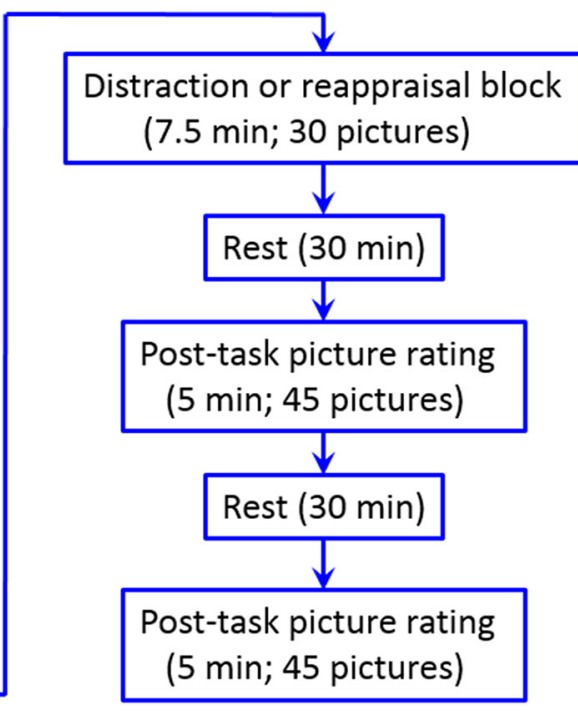

EEG recording

Figure 1. Experimental paradigm and sample images. $\boldsymbol{A}$, Stimulus presentation in one experimental trial. Because of copyright, the people in the sample image are replaced by graduate students from the research group. All four people in this picture gave their consent for this material to appear in academic journals. $\boldsymbol{B}$, Experimental procedure. Transcranial magnetic stimulation (TMS), repetitive TMS (rTMS), electroencephalogram (EEG).

As shown in Figure $1 A$, the trial began with a fixation (lasting $2 \mathrm{~s}$ ), followed by the image presentation for $8 \mathrm{~s}$, during which participants were required to either watch passively (during the no-regulation block) or to downregulate their negative emotions using reappraisal (during the reappraisal block) or to use distraction strategies (during the distraction block). They were then asked to report on the level of negative feelings they experienced on a nine-point scale (with a higher score indicating a higher level of negativity), by clicking the left button on the mouse.

At the start of the no-regulation block, participants were instructed as follows: "In this section, please think about how you would feel in a situation similar to that of the highlighted person in this picture." At the start of the reappraisal block, participants were instructed as follows: "In this section, please imagine a better outcome or find a different explanation of the situation. For example, you could imagine that the group of people who are interacting with one another are talking about something that the person alone is not interested in or the person alone could make some change and join the group very soon. After you re-interpret the nature of this scene, please evaluate how you would feel in this situation if you were the highlighted person in the picture." At the start of the distraction block, participants were instructed as follows: "In this section, please visually attend to the picture while producing unrelated neutral thoughts. For example, think about complex geometric designs or your next study plan. After you distract yourself from the picture, please assign a rating to your feelings in the situation as you were the highlighted person in the picture."

The experimental procedure is shown in Figure $1 B$. Participants underwent three $8 \mathrm{~min}$ long TMS sessions during the experiment, with each TMS session occurring before each block. After the viewing and emotional regulation tasks, participants were allowed to relax for 30 and
60 min before rating the valence of the 90 pictures on a nine-point scale ( 1 representing the most negative valence, 5 for a neutral valence, and 9 for the most positive valence). For each participant, the 30 pictures per condition were randomly assigned to the 30 - or 60 -min valence rating task; that is, participants rated 45 pictures ( 15 per condition) during the 30 -min rating task and the other 45 pictures during the 60 -min rating task. The 45 pictures were randomly presented during these two rating tasks. The 30-min rating session was designed to repeat the result of our previous study (He et al., 2020a), while the 60 -min rating session was designed to explore whether the prolonged-effect of TMS manipulation could be still detected 60 min after the task.

\section{Repetitive TMS (rTMS)}

This study used offline, instead of online, TMS to reduce any side effects that may have impacted participants' task performances (e.g., acoustic noise or muscle twitching). The TMS targets were the rVLPFC and rDLPFC for the two experimental groups. For the sham group, the TMS was targeted at the vertex so as to provide a similar scalp sensation as it did in the other two groups (Hartwright et al., 2016; Masina et al., 2019). A figure-eight-shaped coil was connected to the magnetic stimulator (M-100 Ultimate; Yingchi). The location of the coil was determined with reference to the International 10/20 electroencephalogram system (Klem et al., 1999). The rVLPFC is at the F8, the rDLPFC is at the F4, and the vertex is at the Cz. Each participant's resting motor threshold (rMT) was measured from their motor cortex (the C3), with the intensity being defined as $50 \%$ of the pulses that reliably produced thumb twitches (Schutter and van Honk, 2006). The rTMS was applied at $10 \mathrm{~Hz}$ at $90 \%$ of each participant's rMT. Each 8-min session contained 16 trains, with each train lasting for $3.9 \mathrm{~s}$ (a total of 624 pulses) and which were separated by intertrain intervals of $26.1 \mathrm{~s}$ (Raedt et al., 2010). The TMS- 

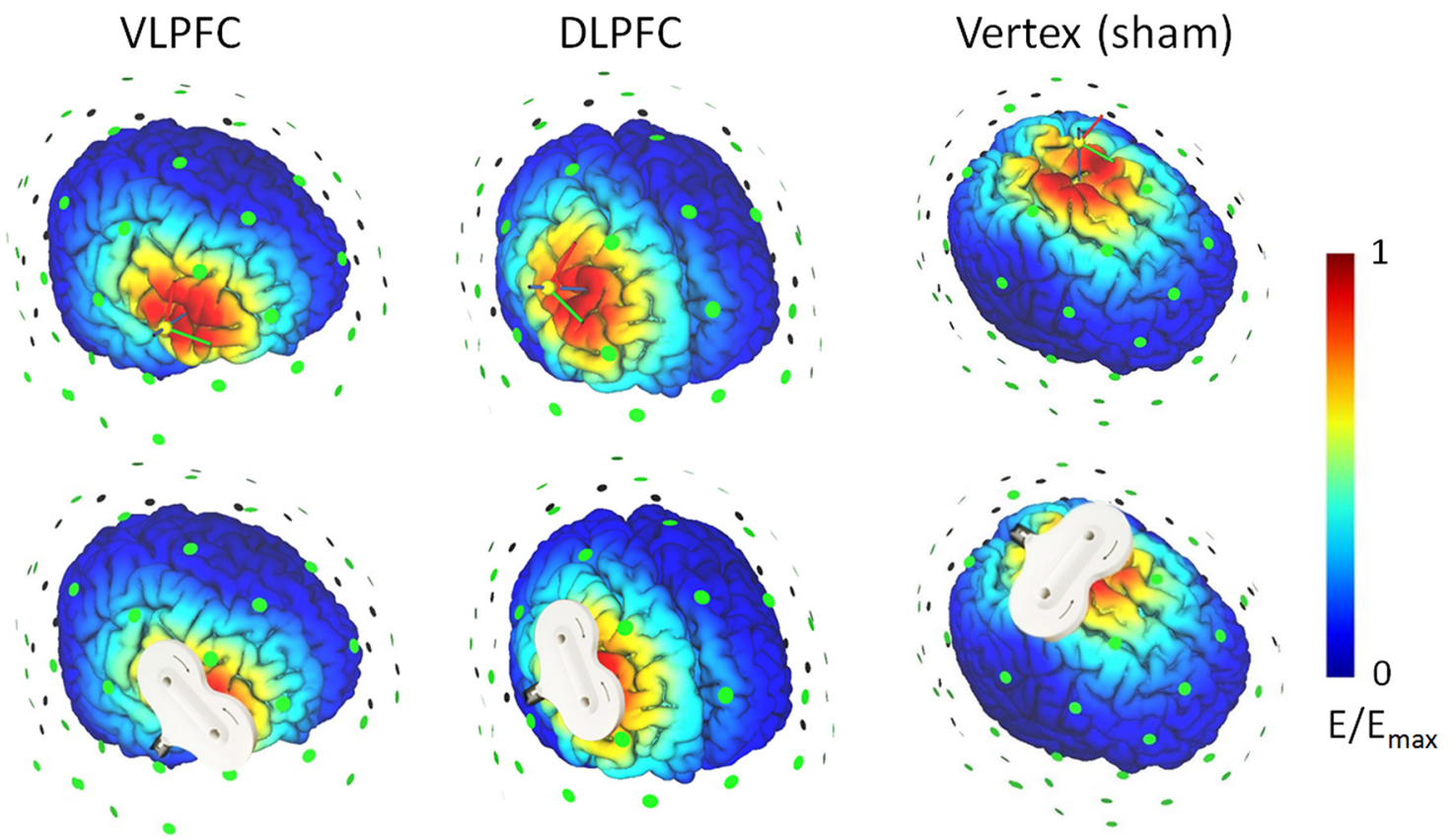

Figure 2. Illustration of TMS electric fields from the three TMS groups. The color represents the electric field strength, scaled from 0 (blue) to the individual maximums (red). The dorsolateral prefrontal cortex (DLPFC) and the ventrolateral prefrontal cortex (VLPFC).

simulated electric field is illustrated on an adult brain model in Figure 2 (SimNIBS; www.simnibs.org).

\section{EEG recordings and analysis}

EEG data were recorded using a 32-channel amplifier (NeuSen.W32, Neuracle), with a sampling frequency of $250 \mathrm{~Hz}$. Electrode impedances were kept below $10 \mathrm{k} \Omega$. The reference electrode was placed at the $\mathrm{CPz}$. No online filter was applied.

Data analysis was performed using MATLAB R2018a (MathWorks). Data were first re-referenced to the average of the left and right mastoids. Ocular artifacts were eliminated using the independent component analysis. Then, the EEG data were filtered using a $0.1-$ to $10-\mathrm{Hz}$ bandpass filter with a slope of $24 \mathrm{~dB} /$ oct. The filtered data were segmented beginning $1 \mathrm{~s}$ before the onset of the picture and lasting for $9 \mathrm{~s}$. The baseline-correction was based on the 1-s prestimulus time window. The Event-related potential (ERP) analysis focused on the late positive potential (LPP; Hajcak and Nieuwenhuis, 2006), which was measured as the average amplitude across the electrode sites at and around $\mathrm{Pz}(\mathrm{P} 3, \mathrm{P} 4, \mathrm{Pz}, \mathrm{CP} 1, \mathrm{CP} 2, \mathrm{POz}, \mathrm{PO}$, and PO4). The time window for the LPP amplitude was chosen according to previous literature (Thiruchselvam et al., 2011; Schönfelder et al., 2014; Paul et al., 2016; Qi et al., 2017), beginning at the end of the typical P3 time window $(500 \mathrm{~ms})$ and lasting for the entire emotional regulation period (500-8000 ms after picture onset). Many previous studies have revealed that the downregulating of negative emotions, including social pain (Wang et al., 2017; He et al., 2020a), reliably reduces the LPP amplitudes (Hajcak et al., 2010; Liu et al., 2012).

\section{Statistics}

Statistical analysis was performed using SPSS Statistics 20.0 (IBM). Descriptive data are presented as mean $\pm \mathrm{SD}$, unless otherwise mentioned. Repeated-measures ANOVAs were performed on the subjective ratings of negative feelings and the LPP amplitudes, with regulation type (no-regulation, reappraisal, or distraction) as the within-subject factor and TMS group (VLPFC, DLPFC, or sham) as the between-subject factor. When analyzing the results of the pictures' valence ratings, a repeated-measures ANOVA was performed with testing time (30 or 60 min after the emotional regulation task) as the within-subject factor and TMS group as the between-subject factor. The Greenhouse-Geisser correction for the ANOVA tests was used whenever appropriate. A twotailed Pearson's correlation was performed between the subjective ratings of negative feelings and the LPP amplitudes from the various regulation blocks and from the different groups. Multiple comparisons were corrected using the Bonferroni method.

\section{Results}

\section{Ratings of negative emotions}

The main effect of the regulation type was found to be highly significant $\left(F_{(2,174)}=303, p<0.001, \eta_{\mathrm{p}}^{2}=0.777\right)$ : participants reported fewer negative feelings in the reappraisal $(3.0 \pm 1.1)$ and distraction blocks $(3.0 \pm 1.0)$ when compared with the passive view (no-regulation) block $(5.6 \pm 1.1$, ps $<0.001)$, whereas the negative feelings between the reappraisal and distraction blocks did not differ $(p=1.000)$. Additionally, there was a significant main effect of the TMS group $\left(F_{(2,87)}=7.08, p=0.001, \eta_{\mathrm{p}}^{2}=0.140\right)$ : participants reported fewer negative feelings in the VLPFC $(3.7 \pm 1.6, p=0.007)$ and DLPFC groups $(3.6 \pm 1.6, p=0.003)$ when compared with the sham TMS group $(4.3 \pm 1.6)$, whereas the negative feelings between the two active TMS groups did not differ $(p=1.000)$.

More importantly, we observed a two-way interaction between TMS group $\times$ regulation type $\left(F_{(4,174)}=3.93, p=0.005\right.$, $\eta_{\mathrm{p}}^{2}=0.083$; Fig. $3 A$; Table 2 ). A simple effects analysis indicated that, while participants showed significantly reduced negative feelings in the reappraisal and distraction blocks when compared with the no-regulation one, across the three TMS groups $(p s<0.001)$, the two emotional regulation blocks showed different patterns of negative feelings across all groups. First, participants reported a slightly greater reduction of negative feelings in the reappraisal than in the distraction block in the VLPFC-activated group $\left(F_{(2,86)}=83.9, p<0.001, \eta_{\mathrm{p}}^{2}=0.661\right.$; reappraisal vs distraction $=2.6 \pm 1.2$ vs $3.1 \pm 0.8, p=0.013)$. Second, contrarily, participants reported a slightly greater reduction in negative feelings during the distraction block than during the reappraisal one in the DLPFC-activated group $\left(F_{(2,86)}=97.4, p<0.001, \eta_{\mathrm{p}}^{2}=0.694\right.$; reappraisal vs distraction $=3.0 \pm 1.1$ vs $2.5 \pm 0.9, p=0.014$ ). Third, no significant difference was observed between the reappraisal and distraction blocks for the sham group $\left(F_{(2,86)}=90.7, p<0.001, \eta_{\mathrm{p}}^{2}=\right.$ 0.678 ; reappraisal vs distraction $=3.6 \pm 1.0$ vs $3.3 \pm 1.1, p=1.000$ ). 

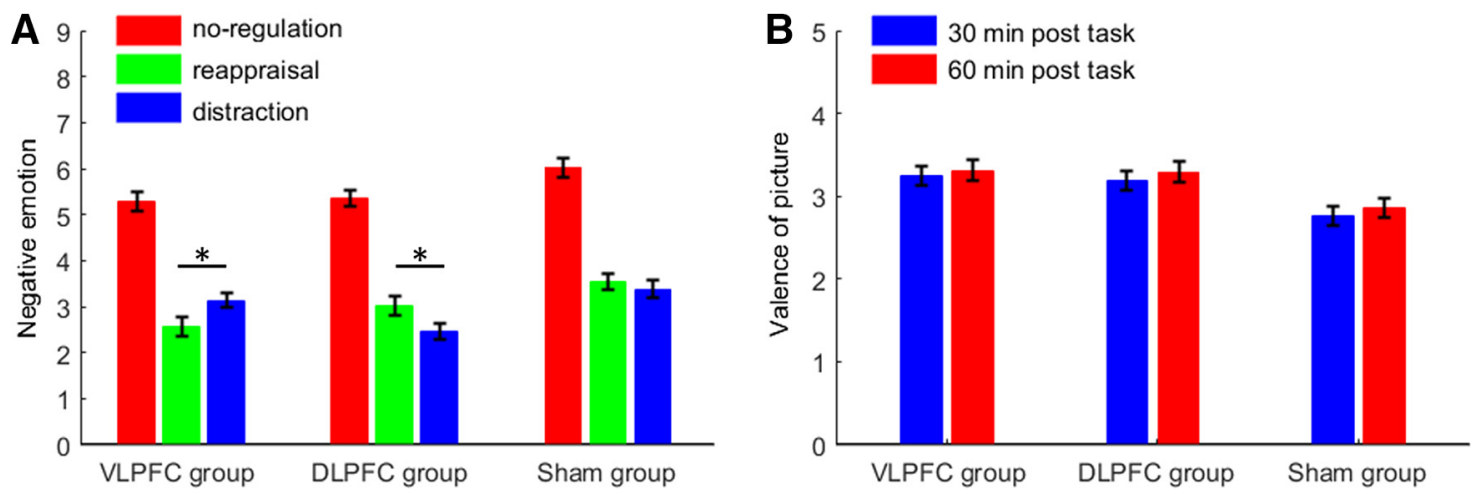

Figure 3. Ratings of negative emotions and picture valences. $A$, Subjective ratings of negative emotions during the emotional regulation task. A nine-point scale was used, with higher scores indicating higher levels of negative emotions. $\boldsymbol{B}$, Postregulation ratings of the pictures' valences. A nine-point scale was used, with higher scores indicating more positive feelings toward the pictures (1 for most negative and 9 for most positive). Bars represent SEM; $* p<0.05$.

Table 2. Descriptive statistics of negative ratings and amplitudes of LPP component (mean \pm SD)

\begin{tabular}{lllll}
\hline Measure & TMS group & No-regulation & Reappraisal & Distraction \\
\hline Negative emotion & VLPFC & $5.28 \pm 1.13$ & $2.57 \pm 1.16$ & $3.13 \pm 0.81$ \\
& DLPFC & $5.35 \pm 0.93$ & $3.02 \pm 1.07$ & $2.46 \pm 0.96$ \\
Sham & $6.03 \pm 1.14$ & $3.54 \pm 1.02$ & $3.38 \pm 1.09$ & \\
LPP amplitude $(\mu V))$ & VLPFC & $3.29 \pm 2.92$ & $0.72 \pm 3.31$ & $2.15 \pm 2.92$ \\
& DLPFC & $3.24 \pm 2.68$ & $2.05 \pm 3.08$ & $0.67 \pm 3.28$ \\
& Sham & $3.52 \pm 2.40$ & $1.92 \pm 2.84$ & $2.34 \pm 3.14$ \\
\hline
\end{tabular}

\section{LPP amplitudes}

The main effect of regulation type was found to be highly significant $\left(F_{(2,174)}=42.4, p<0.001, \eta_{\mathrm{p}}^{2}=0.328\right)$ : participants showed smaller LPP amplitudes in the reappraisal $(1.6 \pm 3.1 \mu \mathrm{V})$ and distraction blocks $(1.7 \pm 3.2 \mu \mathrm{V})$ when compared with the no-regulation one $(3.4 \pm 2.6 \mu \mathrm{V}$, ps $<0.001)$, whereas the LPP amplitudes between the reappraisal and distraction blocks did not differ $(p=1.000)$. The main effect of the TMS group was not found to be significant $(F<1)$.

More importantly, we observed a two-way interaction between TMS group $\times$ regulation type $\left(F_{(4,174)}=7.73, p<0.001, \eta_{\mathrm{p}}^{2}=0.151\right.$; Fig. $4 A, B$; Table 2). The simple effects analysis indicated that, while participants showed reduced LPP amplitudes in the reappraisal and distraction blocks when compared with the no-regulation one across the three TMS groups ( $p s \leq 0.020)$, the two emotional regulation blocks showed differing patterns of LPP amplitudes across all three groups. First, participants showed smaller LPP amplitudes in the reappraisal than in the distraction block in the VLPFC-activated group $\left(F_{(2,86)}=29.9\right.$, $p<0.001, \eta_{\mathrm{p}}^{2}=0.410$; reappraisal vs distraction $=0.7 \pm 3.3 \mathrm{vs}$ $2.2 \pm 2.9 \mu \mathrm{V}, p<0.001)$. Second, contrarily, participants showed smaller LPP amplitudes in the distraction than the reappraisal block in the DLPFC-activated group $\left(F_{(2,86)}=19.4, p<0.001, \eta_{\mathrm{p}}^{2}=0.311\right.$; reappraisal vs distraction $=2.0 \pm 3.1$ vs $0.7 \pm 3.3 \mu \mathrm{V}, p=0.001$ ). Third, no significant differences were observed between the reappraisal and distraction blocks in the sham group $\left(F_{(2,86)}=11.2\right.$, $p<0.001, \eta_{\mathrm{p}}^{2}=0.207$; reappraisal vs distraction $=1.9 \pm 2.8$ vs $2.3 \pm 3.1 \mu \mathrm{V}, p=0.743)$.

\section{Correlations between LPP amplitudes and the subjective} ratings of negative emotions

In each TMS group, we analyzed the correlations between the LPP amplitudes and the ratings of negative emotions in the three regulation blocks, separately. For example, the LPP amplitudes in the reappraisal block were correlated with the rating of
Table 3. Correlation statistics between LPP amplitudes and subjective ratings of negativity $(n=30)$

\begin{tabular}{|c|c|c|c|c|c|c|c|c|c|}
\hline \multirow{2}{*}{$\begin{array}{l}\text { Regulation } \\
\text { type }\end{array}$} & \multicolumn{3}{|c|}{ VLPFC group } & \multicolumn{3}{|c|}{ DLPFC group } & \multicolumn{3}{|c|}{ Sham group } \\
\hline & $r$ & $p$ & $p_{\text {cor }}{ }^{a}$ & $r$ & $p$ & $p_{\text {cor }}{ }^{a}$ & $r$ & $p$ & $p_{\text {cor }}{ }^{a}$ \\
\hline No-regulation & 0.347 & 0.060 & $>0.05$ & 0.297 & 0.110 & $>0.05$ & 0.346 & 60.061 & $>0.05$ \\
\hline Reappraisal & 0.570 & $<0.001$ & $0.009^{* * *}$ & 0.373 & 0.042 & $>0.05$ & 0.251 & 0.182 & $>0.05$ \\
\hline Distraction & 0.338 & 0.068 & $>0.05$ & 0.580 & $<0.001$ & $0.007^{\text {*** }}$ & 0.310 & 0.095 & $>0.05$ \\
\hline
\end{tabular}

negativity in this block for each group $(n=30)$. This procedure produced nine correlations across the three TMS groups (Fig. 4C; Table 3). After correcting for multiple tests, the LPP amplitudes and the ratings of negativity were found to be significantly correlated within the reappraisal block for the VLPFC group $(r=0.570$, corrected $p=0.009)$, as well as during the distraction block for the DLPFC group $(r=0.580$, corrected $p=0.007)$.

\section{Posttask valence ratings of the pictures}

The main effect of the TMS group was found to be significant $\left(F_{(2,87)}=5.6, p=0.005, \eta_{\mathrm{p}}^{2}=0.114\right.$; Fig. $\left.3 B\right)$, the valences reported by both the VLPFC-activated $(3.3 \pm 0.6, p=0.011)$ and the DLPFC-activated groups $(3.2 \pm 0.6, p=0.020)$ were higher than those reported by the sham TMS group $(2.8 \pm 0.6)$. Neither the main effect of testing time $\left(F_{(1,87)}=3.5, p=0.066, \eta_{\mathrm{p}}^{2}=0.038 ; 30\right.$ $\min$ vs $60 \mathrm{~min}=3.1 \pm 0.7$ vs $3.2 \pm 0.7)$ nor the interaction between the TMS group and testing time were significant $(F<1)$.

\section{Discussion}

This study employed TMS to causally explore the neural substrates of explicit emotional regulation, using reappraisal and distraction strategies, within social contexts. We used the subjective rating of negative emotions and the objective ERP index of LPP amplitudes to measure emotion regulation effects. Our results demonstrate that (1) both of the lateral PFC (LPFC) regions within the right hemisphere highly facilitate social pain relief (reflected by lower negative emotional ratings and reduced LPP amplitudes), and (2) while the TMS-activated VLPFC had a better regulation effect during the reappraisal condition, the TMSactivated DLPFC had a better regulation effect during the distraction one.

We focused on the DLPFC and the VLPFC, as they have both been established as key cortical regions involved in emotional 

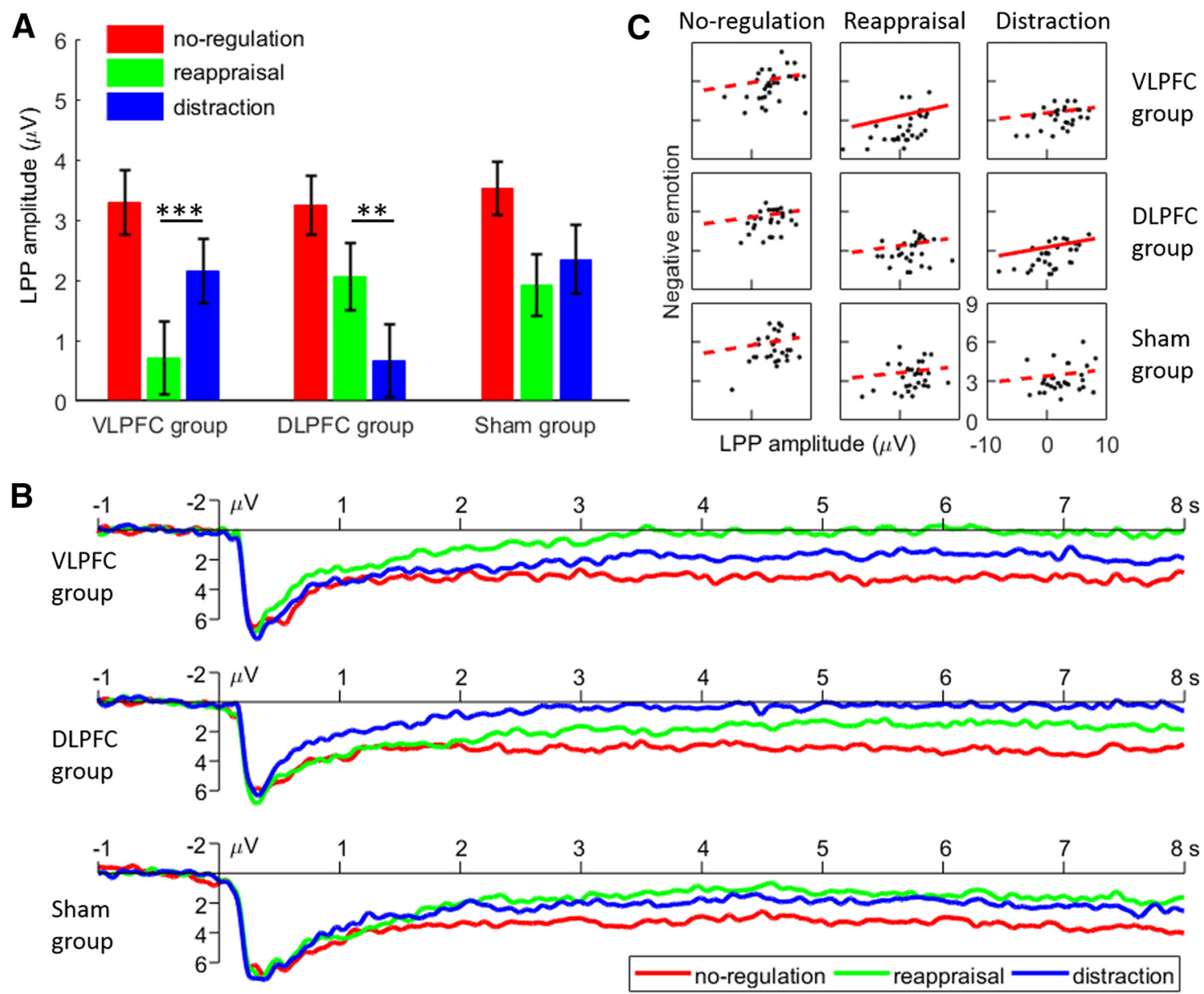

Figure 4. late positive potential (LPP) results. $A$, The LPP amplitudes in the various conditions. Bars represent SEM; $* * p<0.01, * * * p<0.001$. $B$, The grand-mean ERP waveforms for the three TMS groups. The data were averaged across P3, P4, Pz, CP1, CP2, P0z, P03, and P04. C, Correlations between LPP amplitudes and the subjective ratings of negative emotions. While the solid red line indicates a significant correlation, the dashed red line indicates a non-significant correlation.

regulation (Ochsner et al., 2012; Dörfel et al., 2014; Kohn et al., 2014; Morawetz et al., 2017). For one thing, the neural model of emotional processing (Etkin et al., 2015) proposes that, while automatic or implicit emotional regulation predominantly involves the medial PFC (MPFC) system, voluntary or explicit emotional regulation primarily involves the LPFC system. For another, it has been demonstrated that, when compared with the MPFC, the LPFC is more frequently found impaired among psychiatric disorders (Minzenberg et al., 2009; Hamilton et al., 2012) and in individuals experiencing suicidal thoughts (Schmaal et al., 2020). For example, depressed patients tend to show hypoactive LPFCs during voluntary emotional control, whereas their MPFC often functions well during automatic emotional regulation (Rive et al., 2013). These findings suggest that the LPFC, instead of the MPFC, is the ideal target for TMS or neurofeedback therapies aimed at improving psychiatric patients' voluntary emotional regulation abilities. This study contributes to the psychiatric field by causally demonstrating that the two LPFC regions are able to successfully downregulate negative emotions caused by not only non-social but also social events.

Our findings have some potential implications for clinical practice; that is, the right VLPFC/DLPFC regions are valid, and possibly the most direct brain targets, for the treatment of sociallybased emotional dysregulation. Previous studies have shown that, across various clinical populations, patients have often demonstrated reduced recruitment of the VLPFC and the DLPFC during the downregulation of negative emotions (Phillips et al., 2008; Erk et al., 2010; Rive et al., 2013; Zilverstand et al., 2017; Park et al., 2019). More relevant to our work, studies have found that depressed patients demonstrate reduced activation in their right VLPFCs in response to social exclusion images when compared against healthy controls (Elliott et al., 2012), and that enhanced LPFC engagement during negative emotional regulation predicted a decrease in depression severity over six months (Heller et al., 2013). Beyond the findings of these previous studies, our work further reveals the causal role of the VLPFC/DLPFC on social pain relief, which provides a clear rationale for targeting these two areas in the treatment of patients with deficits in social emotional regulation.

The most novel finding of this study is that distraction and reappraisal strategies show double dissociation to a certain extent. This result is in line with the cognitive model of emotional regulation (Gross, 1998), which states that these two regulation strategies function separately during the early and late stages of peoples' cognitive control of their emotions. Additionally, this finding is consistent with previous fMRI observations, demonstrating that distraction and reappraisal, specifically and separately, recruit the DLPFC and the VLPFC, respectively (Dörfel et al., 2014; Moodie et al., 2020). The contribution of this study is two-fold. First, we used a manipulation technique to separate the regulatory functions of the DLPFC and the VLPFC across two groups so as to provide causal evidence beyond that of previous neuroimaging findings. Second, this study found that the LPP amplitudes during the reappraisal and distraction conditions were specifically 
correlated with the negative ratings reported by the VLPFC-activated and the DLPFC-activated groups. These results provide novel electrophysiological evidence for the relative specificity of the VLPFC and the DLPFC during emotional regulation in the use of reappraisal and distraction strategies, respectively.

Previous studies have established that the DLFPC plays a fundamental role in peoples' cognitive control abilities (Crone and Steinbeis, 2017) and is a key region involved in the attentional network (Zwanzger et al., 2014). Given that the core cognitive process of distraction is essentially an attentional shift (Ochsner and Gross, 2005), the DLPFC is frequently implicated as a critical brain region that manages a person's attention during the early stages of emotional regulation (Kohn et al., 2014; Zwanzger et al., 2014). The DLPFC has been shown to indirectly inhibit subcortical limbic structures, such as the amygdala (Park et al., 2019). In particular, the DLPFC communicates with the dorsal ACC/MPFC to monitor conflict and signal the need for behavioral change. The DLPFC also communicates with the parietal region to signal the latter to allocate one's attention elsewhere. These two pathways associated with the DLPFC during emotional regulation result in the downregulation of emotional responses from the ventral ACC/MPFC-amygdala network (Ochsner and Gross, 2005; Mitchell, 2011; Iordan et al., 2019). It has been established that the DLPFC is highly impaired in various psychiatric disorders (Hamilton et al., 2012; Glausier and Lewis, 2018). For example, hypoactivity within this cortical region was observed during attentional control in either emotional regulation or distractor inhibition tasks among patients with depression (Fales et al., 2008) and anxiety (Bishop, 2009).

Unlike the DLPFC, many studies have found that the VLPFC is the primary region involved in reappraisal strategies (Price et al., 2013; Dörfel et al., 2014). It achieves regulatory goals by selecting goal-consistent responses using one's semantic memory and inhibits goal-inconsistent responses so as to reinterpret the affective stimuli (Wager et al., 2008; Ochsner et al., 2009, 2012). In particular, the VLPFC modulates emotional processing through direct projections to the ventral MPFC-amygdala pathway (Silvers et al., 2017), while it also inhibits undesirable responses through projections to both the ACC and the insula (Kohn et al., 2014). Relevant to the current work, a recent study using tDCS demonstrated that successful achievement of emotional reappraisal was based primarily on the VLPFC rather than on the DLPFC (Marques et al., 2018). Taken together, the findings of previous studies in conjunction with our own provide converging evidence that the VLPFC plays a critical role in emotional regulation using reappraisal strategies.

The findings that the VLPFC and the DLPFC play relatively specific roles in different regulation strategies are valuable for clinical practice, which might help to refine the targeting of brain areas in future treatment protocols. For example, anxiety and depressive symptoms are associated with a less frequent use of reappraisal strategies when compared with healthy controls (Dryman and Heimberg, 2018). Meanwhile, the specific strategy that one employs also depends on certain contextual information, for example, distraction is more frequently used for highintensity stimuli, whereas reappraisal is preferred following low-intensity stimuli (Sheppes et al., 2011; Shafir et al., 2015). We thus propose that TMS or neurofeedback therapies should target the most accurate brain region based on each patient's cognitive characteristics, symptoms, or trigger factors so as to effectively enhance their unique emotional regulation abilities.

Another encouraging finding is that the TMS-induced effect of social pain relief persisted for more than $1 \mathrm{~h}$, as revealed by more positive valances reported at both 30 and $60 \mathrm{~min}$ following emotional regulation by the two active TMS groups when compared with the sham group. This result not only corroborates those of previous studies that found that the TMS-activated VLPFC reduces social pain after $0.5 \mathrm{~h}$ following emotional reappraisal (He et al., 2020a), but also extends the prolonged-effect of the TMS in social emotional regulation to at least $1 \mathrm{~h}$. These findings are consistent with those of prior studies demonstrating that the prolonged-effect of a single rTMS session (averaging 20-25 min) usually lasts for 30-60 min (Valero-Cabré et al., 2017). However, we should have a cautious attitude toward the translation of the current finding to a clinical context, because this study only examined healthy people and the prolonged-effect of $1 \mathrm{~h}$ is far from enough in clinics. Future studies should test the prolonged-effect in clinical patients and try to establish multiple-session protocols so as to maintain these TMS-induced neural plastic changes over a longer term.

Finally, it should be noted that we examined imagined, rather than actual, social pain. Although this imagining paradigm has been proven efficient manner in evoking negative emotions and assessing emotional regulation effects (Ochsner et al., 2002, 2004; Wager et al., 2008), it relies on an empathy-based process that might introduce certain confounding factors. Therefore, we strongly encourage future studies to verify these current findings using paradigms evoking "first-hand" social pain; for example, using Cyberball, Island Getaway, or other similar methods. Additionally, this study focused on the right hemisphere of the VLPFC/DLPFC based on the work of previous studies (Ochsner et al., 2012; Morawetz et al., 2017; He et al., 2018, 2020a,b). Future work should test and compare the left versus right hemispheric effects in both healthy individuals and those diagnosed with certain psychiatric disorders. Last but not least, we speculated in this study that a TMS session with $10 \mathrm{~Hz}$ stimuli could activate brain regions according to a majority of TMS literature (Rossi and Rossini, 2004; Dayan et al., 2013). In order to confirm the current conclusion, it is preferred to add another three groups using an inhibited TMS manipulation (e.g., with $1 \mathrm{~Hz}$ ) in future work.

In summary, both the behavioral and electrophysiological results of this study support the hypotheses that both the VLPFC and the DLPFC play causal roles in peoples' explicit emotional regulation of social pain, and that these two regions show relative functional specificity for reappraisal and distraction strategies. In addition, the TMS effect was observed to be sustainable for at least $1 \mathrm{~h}$. These findings pave the way for the accurate targeting of the VLPFC and/or the DLPFC to improve the social functioning and emotional regulation abilities of people within clinical populations.

\section{References}

Achterberg M, van Duijvenvoorde ACK, Bakermans-Kranenburg MJ, Crone EA (2016) Control your anger! The neural basis of aggression regulation in response to negative social feedback. Soc Cogn Affect Neurosci 11:712-720.

Achterberg M, van Duijvenvoorde ACK, van IJzendoorn MH, BakermansKranenburg MJ, Crone EA (2020) Longitudinal changes in DLPFC activation during childhood are related to decreased aggression following social rejection. Proc Natl Acad Sci USA 117:8602-8610.

Beck AT, Steer RA, Brown GK (1996) Beck depression inventory, Ed 2. San Antonio: The Psychological Corporation.

Bishop SJ (2009) Trait anxiety and impoverished prefrontal control of attention. Nat Neurosci 12:92-98.

Buhle JT, Silvers JA, Wager TD, Lopez R, Onyemekwu C, Kober H, Weber J, Ochsner KN (2014) Cognitive reappraisal of emotion: a meta-analysis of human neuroimaging studies. Cereb Cortex 24:2981-2990. 
Crone EA, Steinbeis N (2017) Neural perspectives on cognitive control development during childhood and adolescence. Trends Cogn Sci 21:205-215.

Dayan E, Censor N, Buch ER, Sandrini M, Cohen LG (2013) Noninvasive brain stimulation: from physiology to network dynamics and back. Nat Neurosci 16:838-844.

Davey CG, Allen NB, Harrison BJ, Yücel M (2011) Increased amygdala response to positive social feedback in young people with major depressive disorder. Biol Psychiatry 69:734-741.

Davis MH (1980) A multidimensional approach to individual differences in empathy. J Pers Soc Psychol 10:85.

Dörfel D, Lamke JP, Hummel F, Wagner U, Erk S, Walter H (2014) Common and differential neural networks of emotion regulation by detachment, reinterpretation, distraction, and expressive suppression: a comparative fMRI investigation. Neuroimage 101:298-309.

Downey G, Feldman SI (1996) Implications of rejection sensitivity for intimate relationships. J Pers Soc Psychol 70:1327-1343.

Dryman MT, Heimberg RG (2018) Emotion regulation in social anxiety and depression: a systematic review of expressive suppression and cognitive reappraisal. Clin Psychol Rev 65:17-42.

Durodié B, Wainwright D (2019) Terrorism and post-traumatic stress disorder: a historical review. Lancet Psychiatry 6:61-71.

Eisenberger NI, Lieberman MD, Williams KD (2003) Does rejection hurt? An FMRI study of social exclusion. Science 302:290-292.

Elliott R, Lythe K, Lee R, McKie S, Juhasz G, Thomas EJ, Downey D, Deakin JF, Anderson IM (2012) Reduced medial prefrontal responses to social interaction images in remitted depression. Arch Gen Psychiatry 69:37-45.

Erk S, Mikschl A, Stier S, Ciaramidaro A, Gapp V, Weber B, Walter H (2010) Acute and sustained effects of cognitive emotion regulation in major depression. J Neurosci 30:15726-15734.

Etkin A, Büchel C, Gross JJ (2015) The neural bases of emotion regulation. Nat Rev Neurosci 16:693-700.

Fales CL, Barch DM, Rundle MM, Mintun MA, Snyder AZ, Cohen JD, Mathews J, Sheline YI (2008) Altered emotional interference processing in affective and cognitive-control brain circuitry in major depression. Biol Psychiatry 63:377-384.

Glausier JR, Lewis DA (2018) Mapping pathologic circuitry in schizophrenia. Handb Clin Neurol 150:389-417.

Gross JJ (1998) The emerging field of emotion regulation: an integrative review. Rev Gen Psychol 2:271-299.

Hajcak G, Nieuwenhuis S (2006) Reappraisal modulates the electrocortical response to unpleasant pictures. Cogn Affect Behav Neurosci 6:291-297.

Hajcak G, Macnamara A, Olvet DM (2010) Event-related potentials, emotion, and emotion regulation: an integrative review. Dev Neuropsychol 35:129-155.

Hamilton JP, Etkin A, Furman DJ, Lemus MG, Johnson RF, Gotlib IH (2012) Functional neuroimaging of major depressive disorder: a meta-analysis and new integration of base line activation and neural response data. Am J Psychiatry 169:693-703.

Hartwright CE, Hardwick RM, Apperly IA, Hansen PC (2016) Resting state morphology predicts the effect of theta burst stimulation in false belief reasoning. Hum Brain Mapp 37:3502-3514.

He Z, Lin Y, Xia L, Liu Z, Zhang D, Elliott R (2018) Critical role of the right VLPFC in emotional regulation of social exclusion: a tDCS study. Soc Cogn Affect Neurosci 13:357-366.

He Z, Zhao J, Shen J, Muhlert N, Elliott R, Zhang D (2020a) The right VLPFC and downregulation of social pain: a TMS study. Hum Brain Mapp 41:1362-1371.

He Z, Liu Z, Zhao J, Elliott R, Zhang D (2020b) Improving emotion regulation of social exclusion in depression-prone individuals: a tDCS study targeting right VLPFC. Psychol Med 50:2768-2779.

Heller AS, Johnstone T, Peterson MJ, Kolden GG, Kalin NH, Davidson RJ (2013) Increased prefrontal cortex activity during negative emotion regulation as a predictor of depression symptom severity trajectory over 6 months. JAMA Psychiatry 70:1181-1189.

Hooker CI, Gyurak A, Verosky SC, Miyakawa A, Ayduk Ö (2010) Neural activity to a partner's facial expression predicts self-regulation after conflict. Biol Psychiatry 67:406-413.

Hsu DT, Sanford BJ, Meyers KK, Love TM, Hazlett KE, Walker SJ, Mickey BJ, Koeppe RA, Langenecker SA, Zubieta J-K (2015) It still hurts: altered endogenous opioid activity in the brain during social rejection and acceptance in major depressive disorder. Mol Psychiatry 20:193-200.
Iordan AD, Dolcos S, Dolcos F (2019) Brain activity and network interactions in the impact of internal emotional distraction. Cereb Cortex 29:26072623.

Klem GH, Lüders HO, Jasper HH, Elger C (1999) The ten-twenty electrode system of the international federation. The international federation of clinical neurophysiology. Electroencephalogr Clin Neurophysiol Suppl 52:3-6.

Koban L, Jepma M, Geuter S, Wager TD (2017a) What's in a word? How instructions, suggestions, and social information change pain and emotion. Neurosci Biobehav Rev 81:29-42.

Koban L, Kross E, Woo C-W, Ruzic L, Wager TD (2017b) Frontal-brainstem pathways mediating placebo effects on social rejection. J Neurosci 37:3621-3631.

Koenigsberg HW, Fan J, Ochsner KN, Liu X, Guise K, Pizzarello S, Dorantes C, Tecuta L, Guerreri S, Goodman M, New A, Flory J, Siever LJ (2010) Neural correlates of using distancing to regulate emotional responses to social situations. Neuropsychologia 48:1813-1822.

Kohn N, Eickhoff SB, Scheller M, Laird AR, Fox PT, Habel U (2014) Neural network of cognitive emotion regulation - An ALE meta-analysis and MACM analysis. Neuroimage 87:345-355.

Laceulle OM, Veenstra R, Vollebergh WAM, Ormel J (2017) Sequences of maladaptation: preadolescent self-regulation, adolescent negative social interactions, and young adult psychopathology. Dev Psychopathol 31: 279-292.

Liebowitz MR (1987) Social phobia. Mod Probl Pharmacopsychiatry 22:141173.

Liu Y, Huang H, McGinnis-Deweese M, Keil A, Ding M (2012) Neural substrate of the late positive potential in emotional processing. J Neurosci 32:14563-14572.

Marques LM, Morello LYN, Boggio PS (2018) Ventrolateral but not dorsolateral prefrontal cortex tDCS effectively impact emotion reappraisal effects on emotional experience and interbeat interval. Sci Rep 8:1-12.

Masina F, Tarantino V, Vallesi A, Mapelli D (2019) Repetitive TMS over the left dorsolateral prefrontal cortex modulates the error positivity: an ERP study. Neuropsychologia 133:107153.

Masten CL, Eisenberger NI, Borofsky LA, Pfeifer JH, McNealy K, Mazziotta JC, Dapretto M (2009) Neural correlates of social exclusion during adolescence: understanding the distress of peer rejection. Soc Cogn Affect Neurosci 4:143-157.

Masten CL, Eisenberger NI, Borofsky LA, McNealy K, Pfeifer JH, Dapretto M (2011) Subgenual anterior cingulate responses to peer rejection: a marker of adolescents' risk for depression. Dev Psychopathol 23:283-292.

Minzenberg MJ, Laird AR, Thelen S, Carter CS, Glahn DC (2009) Meta-analysis of 41 functional neuroimaging studies of executive function in schizophrenia. Arch Gen Psychiatry 66:811-822.

Mitchell DGV (2011) The nexus between decision making and emotion regulation: a review of convergent neurocognitive substrates. Behav Brain Res 217:215-231.

Moodie CA, Suri G, Goerlitz DS, Mateen MA, Sheppes G, McRae K, LakhanPal S, Thiruchselvam R, Gross JJ (2020) The neural bases of cognitive emotion regulation: the roles of strategy and intensity. Cogn Affect Behav Neurosci 20:387-407.

Morawetz C, Bode S, Derntl B, Heekeren HR (2017) The effect of strategies, goals and stimulus material on the neural mechanisms of emotion regulation: a meta-analysis of fMRI studies. Neurosci Biobehav Rev 72:111128.

Nishiyama Y, Okamoto Y, Kunisato Y, Okada G, Yoshimura S, Kanai Y, Yamamura T, Yoshino A, Jinnin R, Takagaki K, Onoda K, Yamawaki S (2015) fMRI study of social anxiety during social ostracism with and without emotional support. PLoS One 10:e0127426.

Nolan SA, Flynn C, Garber J (2003) Prospective relations between rejection and depression in young adolescents. J Pers Soc Psychol 85:745-755.

Ochsner KN, Gross JJ (2005) The cognitive control of emotion. Trends Cogn Sci 9:242-249.

Ochsner KN, Bunge SA, Gross JJ, Gabrieli JDE (2002) Rethinking feelings: an FMRI study of the cognitive regulation of emotion. J Cogn Neurosci 14:1215-1229.

Ochsner KN, Ray RD, Cooper JC, Robertson ER, Chopra S, Gabrieli JDE, Gross JJ (2004) For better or for worse: neural systems supporting the cognitive down- and up-regulation of negative emotion. Neuroimage 23:483-499. 
Ochsner KN, Ray RR, Hughes B, Mcrae K, Cooper JC, Weber J, Gabrieli JDE, Gross JJ (2009) Bottom-up and top-down processes in emotion generation: common and distinct neural mechanisms. Psychol Sci 20:13221331.

Ochsner KN, Silvers JA, Buhle JT (2012) Functional imaging studies of emotion regulation: a synthetic review and evolving model of the cognitive control of emotion. Ann NY Acad Sci 1251:E1-E24.

Onoda K, Okamoto Y, Nakashima K, Nittono H, Yoshimura S, Yamawaki S, Yamaguchi S, Ura M (2010) Does low self-esteem enhance social pain? The relationship between trait self-esteem and anterior cingulate cortex activation induced by ostracism. Soc Cogn Affect Neurosci 5:385-391.

Park C, Rosenblat JD, Lee Y, Pan Z, Cao B, Iacobucci M, McIntyre RS (2019) The neural systems of emotion regulation and abnormalities in major depressive disorder. Behav Brain Res 367:181-188.

Paul S, Simon D, Endrass T, Kathmann N (2016) Altered emotion regulation in obsessive-compulsive disorder as evidenced by the late positive potential. Psychol Med 46:137-147.

Phillips ML, Ladouceur CD, Drevets WC (2008) A neural model of voluntary and automatic emotion regulation: implications for understanding the pathophysiology and neurodevelopment of bipolar disorder. Mol Psychiatry 13:833-857.

Price RB, Paul B, Schneider W, Siegle GJ (2013) Neural correlates of three neurocognitive intervention strategies: a preliminary step towards personalized treatment for psychological disorders. Cognit Ther Res 37:657672.

Qi S, Li Y, Tang X, Zeng Q, Diao L, Li X, Li H, Hu W (2017) The temporal dynamics of detached versus positive reappraisal: an ERP study. Cogn Affect Behav Neurosci 17:516-527.

Raedt R, De Leyman L, Baeken C, Schuerbeek P, Van Luypaert R, Vanderhasselt M, Dannlowski U (2010) Neurocognitive effects of HFrTMS over the dorsolateral prefrontal cortex on the attentional processing of emotional information in healthy women: an event-related fMRI study. Biol Psychol 85:487-495.

Riva P, Romero Lauro LJ, DeWall CN, Bushman BJ (2012) Buffer the pain away: stimulating the right ventrolateral prefrontal cortex reduces pain following social exclusion. Psychol Sci 23:1473-1475.

Riva P, Lauro LJR, N, DeWall C, Chester DS, Bushman BJ (2015) Reducing aggressive responses to social exclusion using transcranial direct current stimulation. Soc Cogn Affect Neurosci 10:352-356.

Rive MM, van Rooijen G, Veltman DJ, Phillips ML, Schene AH, Ruhé HG (2013) Neural correlates of dysfunctional emotion regulation in major depressive disorder. A systematic review of neuroimaging studies. Neurosci Biobehav Rev 37:2529-2553.

Rossi S, Rossini PM (2004) TMS in cognitive plasticity and the potential for rehabilitation. Trends Cogn Sci 8:273-279.
Shafir R, Schwartz N, Blechert J, Sheppes G (2015) Emotional intensity influences pre-implementation and implementation of distraction and reappraisal. Soc Cogn Affect Neurosci 10:1329-1337.

Schmaal L, van Harmelen A-L, Chatzi V, Lippard ETC, Toenders YJ, Averill LA, Mazure CM, Blumberg HP (2020) Imaging suicidal thoughts and behaviors: a comprehensive review of 2 decades of neuroimaging studies. Mol Psychiatry 25:408-427.

Schönfelder S, Kanske P, Heissler J, Wessa M (2014) Time course of emotion-related responding during distraction and reappraisal. Soc Cogn Affect Neurosci 9:1310-1319.

Schutter DJ, van Honk J (2006) A standardized motor threshold estimation procedure for transcranial magnetic stimulation research. J ECT 22:176178.

Sheppes G, Scheibe S, Suri G, Gross JJ (2011) Emotion-regulation choice. Psychol Sci 22:1391-1396.

Silvers JA, Insel C, Powers A, Franz P, Helion C, Martin RE, Weber J, Mischel W, Casey BJ, Ochsner KN (2017) VlPFC-vmPFC-amygdala interactions underlie age-related differences in cognitive regulation of emotion. Cereb Cortex 27:3502-3514.

Spielberger CD, Gorsuch RL, Lushene RD (1983) Manual for the state-trait anxiety inventory (form Y). Palo Alto: Consulting Psychologist Press.

Thiruchselvam R, Blechert J, Sheppes G, Rydstrom A, Gross JJ (2011) The temporal dynamics of emotion regulation: an EEG study of distraction and reappraisal. Biol Psychol 87:84-92.

Valero-Cabré A, Amengual JL, Stengel C, Pascual-Leone A, Coubard OA (2017) Transcranial magnetic stimulation in basic and clinical neuroscience: a comprehensive review of fundamental principles and novel insights. Neurosci Biobehav Rev 83:381-404.

Vijayakumar N, Cheng TW, Pfeifer JH (2017) Neural correlates of social exclusion across ages: a coordinate-based meta-analysis of functional MRI studies. Neuroimage 153:359-368.

Vrtička P, Sander D, Vuilleumier P (2011) Effects of emotion regulation strategy on brain responses to the valence and social content of visual scenes. Neuropsychologia 49:1067-1082.

Wager TD, Davidson ML, Hughes BL, Lindquist MA, Ochsner KN (2008) Prefrontal-subcortical pathways mediating successful emotion regulation. Neuron 59:1037-1050.

Wang H, Braun C, Enck P (2017) How the brain reacts to social stress (exclusion) - a scoping review. Neurosci Biobehav Rev 80:80-88.

Zilverstand A, Parvaz MA, Goldstein RZ (2017) Neuroimaging cognitive reappraisal in clinical populations to define neural targets for enhancing emotion regulation. A systematic review. Neuroimage 151:105-116.

Zwanzger P, Steinberg C, Alissa M, Bröckelmann A, Dobel C, Zavorotnyy M, Domschke K, Junghöfer M (2014) Inhibitory repetitive transcranial magnetic stimulation (rTMS) of the dorsolateral prefrontal cortex modulates early affective processing. Neuroimage 101:193-203. 